\title{
Precursor and borderline lesions of the thyroid (indolent lesions of epithelial origin): from theory to practice
}

\author{
Sule Canberk ${ }^{1,2,3}$ \\ ${ }^{1}$ Instituto de Investigação e Inovação em Saúde (i3S), University of Porto, Rua Alfredo Allen, 208 4200-135, Porto, Portugal; ${ }^{2}$ Institute of Molecular \\ Pathology and Immunology of the University of Porto (Ipatimup), Rua Júlio Amaral de Carvalho 45, 4200-135 Porto, Portugal; ${ }^{3}$ Abel Salazar \\ Biomedical Sciences Institute (ICBAS), University of Porto, Rua Jorge de Viterbo Ferreira 228, 4050-313, Porto, Portugal \\ Correspondence to: Sule Canberk, MD, MIAC. I3S, Instituto de Investigação e Inovação em Saúde, University of Porto, Rua Alfredo Allen, $4200-135$ \\ Porto, Portugal. Email: scanberk@ipatimup.pt.
}

\begin{abstract}
The precursor lesions of thyroid neoplasms can arise from either C cells or follicular cells. Although MTC has an established and recognized precursor lesion, the C-cell hyperplasia, the same does not occur for the follicular cell-derived tumors, which are much more frequent. One of the significant obstacles to recognizing follicular cell-derived precursor lesions (FCPL) is the lack of refinement of the morphological spectrum and biology of these putative premalignant lesions. What are the "gold standard" histological criteria in thyroid pathology to identify the progression of dysplasia to cancer? Diagnostical irreproducibility and misnomer in the terminology of some FCPLs have been lying behind the answer to this question. The last past decades' advances in molecular pathology allow us to transform the knowledge in thyroid pathology to cancer prevention and early detection, which will only be possible by improving our understanding of the nature of thyroid precursor and borderline lesions. This review, amassed with the augmented expertise of thyroid pathology documented in the literature, is an attempt at underlining the present understanding of precursor and borderline lesions of the thyroid, with a particular highlight on practice differences in Asian and Western geographies.
\end{abstract}

Keywords: Thyroid gland; follicular cells; precursor lesions; borderline lesions; low-risk carcinomas; indolent lesion of epithelial origin; indolent lesion of epithelial origin (IDLE); overtreatment

Submitted Mar 31, 2020. Accepted for publication Aug 12, 2020.

doi: $10.21037 / g s-20-429$

View this article at: http://dx.doi.org/10.21037/gs-20-429

\section{Introduction}

There is a broad range spectrum of entities classified as a precursor or borderline lesions in the thyroid gland, which in fact encompasses benign, borderline, and lowmalignant lesions. The exact border differentiating these terms is not easy to define in thyroid gland pathology, and "precursor"/"borderline" names have been used as a synonym in the literature on many occasions. In 2014, Esserman et al. (1) suggested the term "indolent lesion of epithelial origin" (IDLE) as referring to indolent/borderline neoplasms and their precursors. The standardized designation and recognition of these lesions differ in various anatomic sites due to the variety of risk factors and pathobiology. On sites where a conventional dysplasia definition exists, these lesions are more readily recognized before the invasion, allowing us to diagnose and understand their nature and biologic behavior. In some organs, such as the uterine cervix, the recognition of true precursor lesions allowed the establishment of successful populationbased screening programs.

Although some developments have been achieved in other organs, including breast, colon, and urinary tract, a significant limitation persists in the unveiling of the natural history of IDLEs in the thyroid. In fact, the concept of dysplasia in the thyroid gland is debatable. In 2013, Chui et al. (2) suggested the term "Follicular epithelial dysplasia" (FED) for a putative preneoplastic lesion of papillary thyroid carcinoma in chronic lymphocytic thyroiditis. 
The term FED was reserved for foci of atypical cells less than $1.0 \mathrm{~mm}$ with moderate cytological atypia and architectural distortion, distinct from the surrounding parenchyma. Although used in other organs (for example, in atypical ductal hyperplasia of the breast), size is not a strong criterium to classify a lesion as dysplastic. Even though the basement membrane (basal lamina) of the thyroid gland is a well-accepted structure standing between follicular cells and interstitial stromal tissue, the lack of conventional "dysplasia" definition has been blurring the boundaries of the precursor lesion and its morphological distinction among IDLEs. Therefore, this distinction has been attempted based on the prognostic clinical outcomes of the different lesions. Since only a few tumors show the unrelenting ability to metastasize and cause patient death, this approach is not entirely unreasonable.

The IDLE proposal covers the majority $(>60 \%)$ of thyroid carcinomas, which are currently classified by the pathologists in the malignant category $(1,3)$. These low-grade tumors that classified in the cancer category can be downgraded to either benign or borderline tumor categories to solve the over-diagnosis and overtreatment in thyroid nodule practice. Recently, Kakudo et al. (3). suggested that this concept can be adopted in the multistep model of follicular cell-derived thyroid carcinogenesis. The same author introduced the concept of "low-risk intrathyroidal neoplasia" which encompasses thyroid follicular cell tumors currently classified in different categories, benign (follicular adenoma), borderline (UMP and NIFTP) and low-risk thyroid follicular cell carcinoma (encapsulated PTC, minimally invasive FTC and papillary microcarcinoma) $(4,5)$. Although differences in risk factors, epidemiology, and clinical management with geographical areas exist, the central common aim for both Asian and Western pathologists is to build a bridge between histopathological classification and clinically relevant risk stratification of thyroid tumors.

Following the advances in the imaging modalities to detect thyroid nodules, the frequency of recognition of these lesions (precursor/borderline) has been increasing in the thyroid, hastening the need for more precise risk stratification methods and effective early intervention $(6,7)$. Understanding the early phases of thyroid carcinogenesis at the molecular and cellular level remains a key challenge, but after the cancer genome atlas (TCGA) comprehensive study on profiling of driver genes for each cancer type, now is the era for unraveling genomic events behind the precancerous lesions (8). This trend ushers in a new collaboration of "Pre-Cancer Genome Atlas (PCGA)," as already called out by some authors in the literature $(9,10)$.

This review, based on the accumulated expertise in the literature of thyroid pathology, is an attempt to provide an outline of the present understanding of IDLEs of thyroidnamely neoplasms with indolent nature and precursor lesions-, with a particular highlight over practice differences in Asian and Western geographies.

\section{What are the IDLES of thyroid?}

IDLEs of thyroid can arise from either C cells or follicular cells. As a precursor lesion of medullary thyroid carcinoma (MTC), the role of $\mathrm{C}$ cell hyperplasia progressing into malignancy has been largely explored, two distinct types of $\mathrm{C}$ cell hyperplasia have been described: "reactive/ physiologic" and "neoplastic" (11). Precursor neoplastic $\mathrm{C}$ cell hyperplasia has generally been documented in the setting of hereditary MTC syndromes. MTC only comprises $5-10 \%$ of all thyroid malignancies, and of these, hereditary MTCs correspond to 25\% (11). Although the follicular cell-derived lesions are much more frequent than the $\mathrm{C}$ cell-derived lesions, the precursor/borderline (IDLE) lesions of follicular epithelial cell origin have remained in the dark until the Chernobyl nuclear power plant accident. In 2000, the Chernobyl Pathologists Group concluded the multistep process of radiation carcinogenesis begins in histologically normal thyroid tissue and may involve dose-dependent gene expression changes $(12,13)$. This began the unveiling of the potential follicular cell-derived precursor lesions (FCPL) through two terminology proposals as "the follicular tumor with uncertain malignant potential" (FT-UMP) and "the welldifferentiated tumor with uncertain malignant potential" (WDT-UMP) (13). This trend led to the introduction of the "non-invasive follicular thyroid neoplasm with papillarylike nuclear features" (NIFTP) terminology in 2016, which was a step in the right direction for refining our understanding of indolent lesions of the thyroid gland (14).

Theoretically, in molecular pathology, neoplasms are originated from the monoclonal proliferation of genetically transformed cells, while hyperplasia stems from a polyclonal proliferation of untransformed cells (15). In 2012, Mete and Asa pointed that the nodules which represent monoclonal proliferation generally are seen solitary (follicular adenoma with or without papillary structure, later called a papillary adenoma), but may also arise on the background of polyclonal proliferation, such as benign nodules in the setting of multi-nodular goiter (colloidal nodule, 
hyperplastic adenomatous nodules with or without papillary hyperplasia) (15). The same authors claimed a spectrum of cytological and architectural changes for small lesions on the background of chronic lymphocytic thyroiditis (CLT), which led the transition from reactive to dysplasia, and from the latter to malignancy (16). To wit, this shows the fact that clonality is not always detectable by histomorphology in the thyroid (15). Through this hypothesis and fact, the authors recommended the term "follicular nodular disease" (FND) instead of "multi-nodular goiter" (MNG), and this article strength the multistep carcinogenesis model in thyroid pathology by highlighting the possibility of being a precursor lesion for all benign follicular nodules regardless the solitary setting (15). Indeed, multifocality is one of the fundamental properties shared by precursor lesions $(17,18)$.

Besides these lesions above, Kakudo et al. (3) have extended FCPL by adding solid cell nests, dyshormonogenetic goiter, HTT (hyalinizing trabecular tumor), FT-UMP, WDTUMP, NIFTP, papillary microcarcinoma, FTC (follicular thyroid carcinoma) with only capsular invasion, encapsulated papillary thyroid carcinoma.

Although with limited evidence in the literature, solid cell nest and its suggested stem cell nature and precursor potential were already issued, as were a few reports of thyroid carcinoma arising in dyshormonogenetic goiter (3).

In the last World Health Organization (WHO) classification, the hyalinizing trabecular adenoma was renamed as a hyalinizing trabecular tumor. It corresponds to an encapsulated tumor characterized by trabecular growth, eosinophilic hyaline substance, and marked PTC-N (PTC nuclear features). Most cases have a benign course, with rare cases showing lymph node metastasis (5).

As can be noticed easily, some of these possible IDLEs are already being classified as "cancer." One of the most debatable entities is the micro PTC (mPTC), as its only relevance stems from the "size." Some observational studies show that most mPTCs no increase in size without progression in terms of tumor stage, suggesting that active surveillance only is an acceptable strategy to manage patients with these tumors $(3,19-23)$. More than $50 \%$ of mPTCs in Japan underwent active surveillance (non-surgical approach), representing only $20 \%$ of the surgically treated thyroid malignancies (24) These tumors are biologically benign after lobectomy alone (100\% survival at ten years) and can be left untreated, probably overlapping significantly with the concept of IDLEs. Indeed, in 2003, following the 12th Annual Porto Cancer Meeting held at the Institute of Molecular Pathology and Immunology of the University of Porto (IPATIMUP), four authors led by Rosai published "The Porto Proposal" (25) for renaming $\mathrm{mPTC}$ as papillary microtumor (PMIT). The rationale behind this proposal shared similar concerns to those of the Asian pathologists, and can be summarized as follows: (I) Rarity of clinically significant $\mathrm{mPTC}$ as a prevalent condition; (II) Sporadic cases of mPTC presenting with metastasis at the time of diagnosis might require additional genetic events to speed up the tumor growth and make them clinically visible. The group underlined that $\mathrm{mPTC}$ is a precursor lesion that may or may not become cancer, belonging to the PTC family (25).

Other lesions not considered malignant, due to their good prognosis in comparison with angioinvasive counterparts, are FTC with capsular invasion and encapsulated PTC, with or without capsular invasion (3).

As a subset of FCPL, "follicular patterned borderline tumors" have always been a subject of heated contention, with a considerable amount of interobserver variation and excellent prognosis. These subsets of tumors have been renamed as "borderline" (precancerous) lesions by Kakudo et al. in 2009 (26). In 2017, Asa reinforced the importance of risk categorization in thyroid follicular neoplasia based on initial phases of carcinogenesis or the progression of premalignant cells to invasive disease, as in the multistep carcinogenesis model (27). Besides the morphological and clinical quests, genomic, molecular dissection has revealed that the biological behavior is strongly affected by RAS- and BRAF-signaling pathways (8). Some authors have advocated that reclassification of PTC into $B R A F^{V 600 E}$-like PTC and RAS-like PTC, merging both the histomorphology and molecular characteristics of the tumor $(28,29)$. These molecular subtypes were strongly associated with prognosis and other distinct clinical characteristics beyond standard histology, suggesting that they can be incorporated into clinical practice. This molecular-based division is supported by the architectural characteristics of the tumor, as RASlike tumors are follicular-patterned, whereas $B R A F^{V 600 E}$-like tumors are papillary $(8,29)$. These results also question the value of PTC-N in the diagnosis of malignancy and may underline the stratified risk approach based on a spectrum of thyroid tumors from benign to borderline to malignant. Recently, Ohba et al. proposed a new borderline tumor entity called NEPRAS ("non-invasive encapsulated papillary RAS-like thyroid tumor") which is characterized by noninvasive encapsulated thyroid lesion with papillary growth and RAS type nuclear changes (30). Later, Rosario (31) published the second case report of NEPRAS, underlying 
the impact on patient management, with the same implications as those accepted for the change from noninvasive encapsulated FV-PTC to NIFTP. This neoplasm is an good example of the cross-sectional transition between RAS and BRAF pathways, which can eventually occur.

\section{What are follicular-patterned borderline tumors of thyroid?}

In the last decade, emerging data revealed the preeminence of (capsular/vascular) invasiveness over the nuclear characteristics of PTC as a criterion for malignancy, and diagnosing these entities became a mélange of both cytologic and architectural components of the tumor. As the ratio of the follicular pattern increases within these tumors, the angst and uncertainty dominate the thyroid pathology practice. Thus, the two new chapters of the $4^{\text {th }}$ edition of the WHO (5) endocrine blue book were dedicated to "other thyroid tumors with an encapsulated follicular pattern" and "NIFTP" (3). As a potential precursor lesion, FT-UMP was accepted in the continuum of non-papillary follicular tumors, between follicular adenoma (FA) and FTC, and WDT-UMP was positioned as a precursor lesion in the continuum of papillary tumor towards to PTC. NIFTP, while sharing the same molecular and architectural profile as FA and FTC family, gives a hand to the PTC family by PTC-N, maybe one more time underline many questions in thyroid pathology that have mentioned in the previous part of the article.

These entities (NIFTP, FT-UMP, WDT-UMP) were not new; the Chernobyl group introduced Uncertain malignant potential (UMP) tumor group in 2000 (13), but it found a place in the WHO classification $4^{\text {th }}$ edition, and encapsulated follicular variant of PTC was always under magnitude with the favorable prognosis among other PTC variants, and its terminology has changed to NIFTP following the advances in molecular pathology (5).

While NIFTP has at once become the single most common source of second opinion cases and the subject of heated debate, tumors of UMP somehow continued to lay in the shadow.

Since both NIFTP and WDT-UMP biologically benign and simple excision is enough in the vast majority of these cases, their distinction is not essential clinically. Following this fact and the continuous debate of the classification of these tumors, Liu et al. analyzed 30 cases for a median follow-up of 80 months and proposed the term WDT$\mathrm{UB}$ (well-differentiated tumors of uncertain behavior), encompassing both WDT-UMP and NIFTP (32).
There has always been considerable interobserver variability in the diagnosis of all these entities, posing a significant challenge in pathology practice. Partly, this might be attributed to the overlapped histopathological findings of both benign and moderate/aggressive malignant tumors in thyroid and insufficient standardization in diagnostic criteria for these borderline, low-risk malignancies.

The question arises why we need to make a diagnosis for these morphologically subtle and subjective borderline lesions when all are biologically low aggressive with a less than $0.2 \%$ metastasis ratio, which is accepted as a mortality prediction (33). However, when addressing a treatable disease, an appropriate measure should not be the mortality rate, but the prevention of over or under treatment due to the diagnostic uncertainties/challenges. Another question is if we should make a differential diagnosis between those three entities-NIFTP, WDT-UMP, and FT-UMP.

\section{Non-invasive follicular thyroid neoplasm with papillary-like nuclear features (NIFTP)}

Many studies revealed the encapsulated/welldemarcated FVPTC has molecular alterations similar to those of follicular adenoma and carcinoma, with lack of BRAF mutations, a high prevalence of RAS mutations, and other RAS-like molecular spectrum mutations/fusions (PAX8/PPARG and THADA fusions, and EIF1AX and BRAF ${ }^{\mathrm{K} 601 \mathrm{E}}$ ) (29). In 2016, a multidisciplinary collaborative group undertook a longterm follow-up (10 to 26 years) of 109 patients and proposed to rename an old entity, corresponding to a subset of encapsulated and non-invasive FVPTC (14). This has been designated as "non-invasive neoplasms", rather than carcinoma. This diagnosis is recommended to be entertained only in the light of strict inclusion and exclusion criteria. The diagnostic term used for these tumors as it stands now is "the non-invasive follicular tumor with papillary-like nuclear features" (NIFTP) (14,34), and patients with NIFTP should be considered eligible for limited initial surgery (lobectomy) alone instead of an extended surgery and radioactive iodine (RAI) therapy (14).

\section{Pathology}

The first proposed criteria to support this diagnosis was defined in the seminal study by Nikiforov et al. (14) in 2016, and included the following: (I) encapsulation or clear demarcation; (II) follicular growth pattern with less than 
$1 \%$ papillae, no psammoma bodies, and no more than $30 \%$ solid/ trabecular/insular growth pattern; (III) nuclear score 2 to 3; (IV) no vascular or capsular invasion; (V) no tumor necrosis; and (VI) no high mitotic activity (defined as at least 3 mitoses per 10 high-power fields). However, many cases with regional lymph node metastases, as well as $B R A F^{V 600 E}$ mutations or high-risk TERT promoter mutations, started to be reported in the literature, mostly owing to misevaluation of "less than $1 \%$ papillae" criterion (35-37). In 2018, the group revisited the criteria for more appropriate segregation of real NIFTP cases, ensuring the diagnose of a targeted subset of tumors that would consistently have a favorable outcome (38). Specific consideration was given to strength the following four criteria; one, papillae (the criterion of $1 \%$ of papillae was modified to no well-formed papillae). Two, sufficient sections needs to be submitted (sampling for entire of the capsule and whole tumor to exclude papillae). Three, molecular characteristics (in the presence of mutations characteristic of conventional PTC or high-risk cancer including $B R A F^{V 600 E}$, RET/PTC, or TERT the diagnosis of NIFTP should not be performed). Lastly, four, fully developed PTC-N (special effort should be spent to exclude the presence of papillae in the potential NIFTP cases with fully developed PTC-N) $(38,39)$. The nuclear scoring system was designed to define the minimum nuclear features of PTC, calling a tumor NIFTP in confidence. The nuclear features of NIFTP were grouped into 3 categories, suggesting a 3 -point scoring scheme, where 0 or 1 is diagnostic of a benign nodule and a score of 2 or 3 is diagnostic of NIFTP: (I) size and shape (nuclear enlargement/ overlapping/ crowding, elongation); (II) nuclear membrane irregularities (irregular contours, grooves, pseudo inclusions); and (III) chromatin characteristics (clearing with margination/glassy nuclei) $(38,39)$. However, even using the nuclear scoring system in pathology specimens reported, at best, an average interobserver variation among authors. The threshold for sufficient nuclear features of PTC has remained a point of the critic, both in the literature and in daily practice, and it also explains the main difference in Asian and Western thyroid practice. One of the first studies on the Western side of the world assessing the interobserver variation in nuclear features was performed through an education module with a pre-evaluation teaching guide of the diagnostic criteria of NIFTP of 30 cases for 76 participants, and it showed an excellent agreement (40). However, the study used still images per case, which, as a corollary, was far from the reflections of real-life complexity. A similar study designed by the Asian Thyroid Working Group ended with a moderate agreement (41). The study was designed without an education module on virtual slides of 30 non-invasive follicular patterned cases evaluated by 9 pathologists. The interobserver agreement for the nuclear grading score was moderate, which may reflect the challenge of daily practice.

Another difference between the two geographies was the prevalence of this controversial entity. A metaanalysis and a retrospective study by the Asian Thyroid Working Group documented that the incidence of NIFTP and FVPTC in the Asian population is lower than those observed in non- Asian series and various interpretations of diagnostic thresholds for PTC probably contributed for such difference $(42,43)$. The study of Bychkov et al. evaluating 26,604 cases from six Asian countries showed a lower rate of NIFTP in Asian countries (1.6\%) when compared with non-Asian countries (13.3\%), and one of the most important reasons for this difference stems from the recognition of the nuclear features (42).

Another challenge of diagnosing NIFTP is the criteria for diagnosing capsular invasion in follicular neoplasms in general, which is still subject to a high interobserver variation.

In our daily practice observing consultation cases, this is one of the most common reasons for the submitted cases to being classified as NIFTP while they actually are WDT-UMP.

The correct diagnosis of NIFTP is only possible when both revised inclusion and exclusion criteria are entirely met. If not, we might be misdiagnosing WDT-UMP or encapsulated PTC as NIFTP. The diagnostic category of NIFTP is beneficial to prevent overtreatment of the cases when diagnosing PTC; however, ignoring WDTUMP might also cause under-diagnosing when tumors are classified as NIFTP. The gap exists not only for those cases suffering issues in the nuclear threshold but also in the evaluation of capsule and vascular invasion, the latter being the most common controversy in thyroid pathology. Until WDT-UMP cases gain the same attention as NIFTP does, the accumulation of correct data from NIFTP cases in the literature will remain as a source of contention. There are still many unclear aspects in NIFTP, including if it can be diagnosed in tumors under $1 \mathrm{~cm}$ or if NIFTP can be oncocytic $(44,45)$. All this taken together may compromise the refinement of NIFTP characterization and the answer to still open questions in NIFTP in future studies.

\section{Cytology}

Renaming non-invasive encapsulated FV-PTC strongly 
affectd the primary output of the diagnostic categories of The Bethesda system for reporting thyroid cytopathology (TBSRTC) (46). Various institutions from all over the world have performed retrospective studies, mainly focusing on the risk of malignancy (ROM) of each category of TBSRTC having NIFTP no longer considered a malignant entity $(43,47-49)$. Large evidence from many institutions indicated reduced requirements for surgical resections as the results revealed the fact that cases of NIFTP have generally been placed under the indeterminate and suspicious categories [atypia of undetermined significance/follicular lesion of undetermined significance (AUS/FLUS), follicular neoplasm/suspicious for follicular neoplasm (FN/SFN) and suspicious for malignancy (SFM)] of TBSRTC. These findings are reflected in the second edition of TBSRTC with the changes in the implied ROM for the indeterminate categories, with a ROM of 6-18\% for AUS/FLUS, $10-40 \%$ for FN/SFN, and $45-60 \%$ for SFM (46). Unfortunately, the cytological diagnosis of NIFTP is not possible at this moment. Many authors accepted the presence of PTC-like nuclear features but highlighted the need to quantify these alterations. The question is not precisely the presence or absence of PTC nuclear features, but the degree of these features representing the nuclear scoring for the standardization of the cytological diagnosis of NIFTP (34). As Seethala et al. (50) noticed, evaluating nuclear features of PTC as a "core diagnostic feature" of NIFTP should compare with the adjacent healthy thyroid tissue, which is not possible for cytology samples. Thus, the creation of a diagnostic algorithm to predict NIFTP by fine-needle aspiration biopsy (FNAB) has been proposed by cytopathologists (51). However, most studies supporting this proposal suffered from insufficient reproducibility. The most robust morphological feature in cytology is the "microfollicular pattern," but it is far from being a "core" characteristic in diagnoses as it encompasses a large spectrum of entities, from benign to malignant. As well-known, the nuclear morphology may frequently mimic some nuclear features of NIFTP in Hashimoto's thyroiditis, functional disorders, prolonged treatment, and artefactual reasons. For this reason, a multidisciplinary approach to diagnosing NIFTP, including image findings in conjunction with molecular tests, still seems to be the best solution for cytology materials.

\section{Tumors of uncertain malignant potential (T-UMPS)}

In 2000, the term "uncertain malignant potential" was suggested by the Chernobyl pathologist group for encapsulated follicular-patterned lesions, due to the gradual extension to malignancy of these biologically low aggressive tumors (13). WDT-UMP and FT-UMP, in their definition, have been suggested to be borderline lesions $(13,52)$. They are pure follicular-patterned neoplasms with questionable capsular or vascular invasion, the difference being defined based on the PTC-N: If the neoplasm represents fully or questionable PTC nuclear features, it should be classified as WT-UMP, whereas it is compatible with FT-UMP if there is an absence of PTC nuclear features. The term well-differentiated tumors of uncertain malignant potential (WDT-UMP) was proposed for morphologically noninvasive or questionable invasive encapsulated follicular tumors with questionable PTC-N (13).

This term still has not taken root in daily clinical practice since it was first introduced to thyroid pathology. Chetty elegantly criticized (53) when saying, "perhaps partly because the term suggests that we as pathologists are 'uncertain' of what we are diagnosing". Recently, Asa (27) reinforced the cogent argument when reasoning "these diagnostic categories invite the freedom to equivocate and leave clinicians and patients with no answers as well as lack of confidence in pathology." Indeed, unlike NIFTP, there are still no tailored guidelines for UMP lesions. Obviously, there is a high possibility that this lack of confidence and the absence of well-designed large cohort studies in the literature to support the standardization in diagnosing WTUMP may be curtailed, and these cases may be shifted to the NIFTP basket.

\section{WDT-UMP}

The term well-differentiated tumors of uncertain malignant potential (WDT-UMP) was proposed for morphologically non-invasive or questionable invasive encapsulated follicular tumors, with questionable PTC-N (13). The cases we now diagnose as NIFTP were a fraction of the WDT-UMP cases. However, after the $4^{\text {th }}$ edition of WHO, WDT-UMP cases are only reserved for the ones composed of welldifferentiated follicular cells having equivocal capsular/ vascular invasion, with questionable PTC-N (5).

In comparison with the recently renamed entity NIFTP, there are quiet limited number of articles in the literature about WDT-UMP. Furthermore, the documented articles before 2017 generally encompass NIFTP cases, since this entity was a fraction of WDT-UMP based on the original description of Chernobyl group. Thus, these lesions were not well-characterized from a molecular, 
morphological, and clinical standpoint. In 2002, Fusco et al. analyzed a group of WDT-UMP cases (WDT-UMP and encapsulated FV-PTC) and concluded that RET activation was in concordance with areas of tumors with cytological alterations, indicating that these areas may lead to the development of invasive PTC (54). Due to the original description of WDT-UMP in 2000, neglecting NIFTP as currently described, data on the actual prevalence of this entity have also remained dubious. Following the authors' suggestion that cases with diffuse equivocal or patchy PTC-N are not malignant and should be included in the "borderline category." This persistent doubt of Asian pathologists about the widely accepted fact that PTC-N are a golden standard of malignancy gave rise to a much broader extension of studies about borderline/precursor lesions and stem cells of thyroid in comparison with the Western side of the world. However, several terminologies and different definitions were used to address borderline lesions. Of those, "well-differentiated tumor of uncertain behavior" (WD-UB), which was suggested by Kakudo and his group, deserves attention $(32,55)$.

The terminology mainly originates from the aforementioned scepticism about making a differential diagnosis among precursor/borderline lesions of thyroid, being described as "is composed of encapsulated tumours with or without nuclear features of PTC and with or without minimal capsular invasion, which includes encapsulated common type PTC, encapsulated follicular variant of PTC, WDT- UMP, minimally invasive and capsular invasion only type FTC and FT-UMP in WHO classification and Williams classification" (55). As can be previous observed, none of the earlier studies in the literature exactly overlap the current diagnostic criteria of WDT-UMP suggested by WHO in 2017. WDTUMP deserves more studies on the renewed criteria, to understand mechanisms of progression to carcinoma and risk stratification, and thereby define clear management of borderline lesions. One of the most recent studies in the literature, which analyzed 150 patients with follicular thyroid tumors including FTA $(\mathrm{n}=48)$, FT-UMP $(\mathrm{n}=32)$, WDT-UMP $(\mathrm{n}=17)$ and FTC $(\mathrm{n}=53)$, found no $B R A F^{V 600 E}$ mutation in WDT-UMP, whereas H/N/K-RAS mutations were positive in the group of cases of WDT-UMP (56). In the Duan et al. study, the authors concluded that WDTUMP might be genetically similar to NIFTP (56). Survival outcomes were available in 94\% FTA (45/48), 100\% WDTUMP (17/17), 91\% FT-UMP (29/32) and 98\%FTC (52/53) cases. Only a few FTC patients presented with initial bone metastasis, disease persistence, recurrence, and died due to disease, whereas none of these were reported in FTA, FTUMP or WDT-UMP group of patients.

Cytologically, the same challenge exists, although most of the cases were placed in the indeterminate categories of TBSRTC as, to a certain extent, they share PTC-N with other lesions. Currently, there are no criteria for the FNA samples of WDT-UMP. Previous studies also suffer from the bias of the difference between previous and current definitions of the entity. Future studies are needed to refine diagnostic criteria of this neglected entity to increase our experience, and this will hopefully lead to better stratification and improved guidelines for a better standardized patient management.

\section{FT-UMP}

The term "follicular tumor of uncertain malignant potential" (FT-UMP) has been proposed for the encapsulated tumors which composed of well-differentiated follicular cells with questionable capsular invasion, and no blood vessel invasion nor PTC-N by Williams et al. However, this group of tumors was re-defined by WHO classification as encapsulated tumors composed of well-differentiated follicular cells with equivocal capsular/vascular invasion without PTC-N. Studies focused on this entity are even fewer when compared to WDT-UMP and NIFTP groups. In daily practice, some of these cases are being misinterpreted as "FTC with minimal capsule invasion" due to the controversial judgment of capsule invasion among pathologists. As underlined in the recent study of Zhu et al., a wide range of interobserver variation in the interpretation of capsular invasion contributes to the challenge in diagnosing in FT-UMP and all encapsulated follicular patterned tumors (57). In the aforementioned study of Duan, and agreement with previous studies, no TERTp mutations were found in patients with FT-UMP or FTA (56). Although TP53 mutations were more frequent in FTUMP than in FTC, the differences were not statistically significant. TSHR mutations were found in FT-UMP, besides HRAS/ NRAS and EIF1AX mutations, which were also detected in a small percentage of FT-UMP cases (56). However, on the contrary of Duan's group, Paulsson et al. (58) analyzed 33 patients with FT-UMP, and 39\% of these were found to harbor TERT mutation. The authors pointed to their in-house protocol for screening confirmed FT-UMP cases for TERT promoter mutations implemented since 2014 as part of the routine clinical 
workup. Based on the results, these cases have been discussed at multidisciplinary conferences in which the TERT promoter mutational screening might motivate more aggressive treatment options than the standardized protocol, where patients are routinely followed for 6 months. These two articles underline once more the need for further documentation focusing on both UMP tumors, to provide standardized and clear guidelines and decreasing individual $a d-h o c$ approach, thereby drawing evidence-based conclusions on how to handle this group of patients.

Cytologically, these cases are expected to fall either in the "suspicious for follicular neoplasms /follicular neoplasm" (SFN/ FN) category or in the "follicular lesion of indeterminate significance" (FLUS) category of TBSRTC, depending on the cellularity of the specimen. Despite the lack of a sufficiently sized study, the prognosis is known to be excellent as in WDT-UMP.

\section{Conclusion and future directions}

The precursor lesions of thyroid neoplasms can arise from either C cells or follicular cells. Although MTC has a wellestablished and recognized pathology lesion - the C-cell hyperplasia-, the same does not occur for the precursor of the follicular cell-derived tumors, which are much more frequent. One of the significant problems in recognizing the FCPLs is the absence of a clear demarcation that differentiates precancerous from cancerous lesions. However, the identification of these lesions as a precursor of malignant tumors will be beneficial in reducing the over-diagnosis and over-treatment of patients with thyroid nodules.

The endeavor of developing and implementing more rational guidelines and screening programs in thyroid pathology, reducing the risks of potential harm for individuals, requires more attention to potential precursor lesions of thyroid as a critical concept to understanding thyroid pathology.

\section{Acknowledgments}

Funding: Through FCT - Fundação para a Ciência e a Tecnologia - in the framework of a PhD grant to SC (SFRH/ $\mathrm{BD} / 147650 / 2019)$.

\section{Footnote}

Provenance and Peer Review: The Guest Editor commissioned this article (Kennichi Kakudo) for the series "Asian and Western Practice in Thyroid Pathology: Similarities and Differences" published in Gland Surgery. The article was sent for external peer review organized by the Guest Editor and the editorial office.

Conflicts of Interest: The author has completed the ICMJE uniform disclosure form (available at http:// dx.doi.org/10.21037/gs-20-429). The editorial office commissioned the series "Asian and Western Practice in Thyroid Pathology: Similarities and Differences" without any funding or sponsorship. The author has no other conflicts of interest to declare.

Ethical Statement: The author is accountable for all aspects of this work. They have ensured that questions related to the accuracy or integrity of any part of the work are appropriately investigated and resolved.

Open Access Statement: This is an Open Access article distributed in accordance with the Creative Commons Attribution-NonCommercial-NoDerivs 4.0 International License (CC BY-NC-ND 4.0), which permits the noncommercial replication and distribution of the article with the strict proviso that no changes or edits are made and the original work is properly cited (including links to both the formal publication through the relevant DOI and the license). See: https://creativecommons.org/licenses/by-nc-nd/4.0/.

\section{References}

1. Esserman LJ, Thompson IM, Reid B, et al. Addressing overdiagnosis and overtreatment in cancer: a prescription for change. Lancet Oncol 2014;15:e234-42.

2. Chui MH, Cassol CA, Asa SL, et al. Follicular epithelial dysplasia of the thyroid: morphological and immunohistochemical characterization of a putative preneoplastic lesion to papillary thyroid carcinoma in chronic lymphocytic thyroiditis. Virchows Arch 2013;462:557-63.

3. Kakudo K, Wakasa T, Kakudo M, et al. Borderline and 
Precursor Lesions of Thyroid Neoplasms: A Missing Link. J Basic Clin Med 2015;4:2-7.

4. Kakudo K, Bychkov A, Bai Y, et al. The new 4th edition World Health Organization classification for thyroid tumors, Asian perspectives. Pathol Int 2018;68:641-64.

5. Lloyd RV, Osamura YR, Kloppel G, Rosai J, editors. Tumours of thyroid gland. WHO Classification of Tumours of Endocrine Organs. 4th ed. Lyon, France: World Health Organization; 2017:78-80.

6. Cramer JD, Fu P, Harth KC, et al. Analysis of the rising incidence of thyroid cancer using the Surveillance, Epidemiology and End Results national cancer data registry. Surgery 2010;148:1147-52.

7. Vigneri R, Malandrino P, Vigneri P. The changing epidemiology of thyroid cancer: why is incidence increasing? Curr Opin Oncol 2015;27:1-7.

8. Cancer Genome Atlas Research Network. Integrated genomic characterization of papillary thyroid carcinoma. Cell 2014;159:676-90.

9. Ryan BM, Faupel-Badger JM. The hallmarks of premalignant conditions: a molecular basis for cancer prevention, Semin Oncol 2016; 43:22-35.

10. Campbell JD, Mazzilli SA, Reid ME, et al. The Case for a Pre-Cancer Genome Atlas (PCGA). Cancer Prev Res (Phila) 2016;9:119-24.

11. Scognamiglio T. C Cell and Follicular Epithelial Cell Precursor Lesions of the Thyroid. Arch Pathol Lab Med 2017;141:1646-52.

12. Abend M, Pfeiffer RM, Ruf C, et al. Iodine-131 dosedependent gene expression: alters in both normal and tumour thyroid tissues of post-Chernobyl thyroid cancers. Br J Cancer 2013;109:2286-94.

13. Williams ED. Guest Editorial: Two Proposals Regarding the Terminology of Thyroid Tumours. Int J Surg Pathol 2000;8:181-3.

14. Nikiforov YE, Seethala RR, Tallini G, et al. Nomenclature revision for encapsulated follicular variant of papillary thyroid carcinoma: a paradigm shift to reduce overtreatment of indolent tumours. JAMA Oncol 2016;2:1023-9.

15. Mete O, Asa SL. Pitfalls in the diagnosis of follicular epithelial proliferations of the thyroid. Adv Anat Pathol 2012;19:363-73.

16. Mete O, Asa SL. Precursor lesions of endocrine system neoplasms. Pathology 2013;45:316-30.

17. Sporn MB. Approaches to prevention of epithelial cancer during the preneoplastic period. Cancer Res 1976;36:2699-702.
18. Canberk S, Montezuma D, Taştekin E, et al. "The other side of the coin": understanding noninvasive follicular tumour with papillary-like nuclear features in unifocal and multifocal settings. Hum Pathol 2019;86:136-42.

19. Ito Y, Miyauchi A. A therapeutic strategy for incidentally detected papillary microcarcinoma of the thyroid. Nat Clin Pract Endocrinol Metab 2007;3:240-8.

20. Arora N, Tubendian HK, Kato MA, et al. Papillary thyroid carcinoma and microcarcinoma: is there a need to distinguish the two? Thyroid 2009;19:473-7.

21. Sugitani I, Toda K, Yamada K, et al. Three distinct kinds of papillary thyroid microcarcinoma should be recognized: our treatment strategies and outcomes. World J Surg 2010;34:1223-31.

22. Miyauchi A. Clinical trials of active surveillance of papillary microcarcinoma of the thyroid. World J Surg 2016;40:516-22.

23. Kakudo K. How to handle borderline/precursor thyroid tumours in management of patients with thyroid nodules. Gland Surg 2018;7:S8-S18.

24. Sugitani I, Ito Y, Miyauchi A, et al. Active Surveillance Versus Immediate Surgery: Questionnaire Survey on the Current Treatment Strategy for Adult Patients with LowRisk Papillary Thyroid Microcarcinoma in Japan. Thyroid 2019;29:1563-71.

25. Rosai J, LiVolsi VA, Sobrinho-Simoes M, et al. Renaming papillary microcarcinoma of the thyroid gland: the Porto proposal. Int J Surg Pathol 2003;11:249-51.

26. Kakudo K, Bai Y, Katayama S, et al. Classification of follicular cell tumours of the thyroid gland: analysis involving Japanese patients from one institute. Pathol Int 2009;59:359-67.

27. Asa SL. The evolution of differentiated thyroid cancer. Pathology 2017;49:229-37.

28. Rivera M, Ricarte-Filho J, Knauf J, et al. Molecular genotyping of papillary thyroid carcinoma follicular variant according to its histological subtypes (encapsulated vs infiltrative) reveals distinct BRAF and RAS mutation patterns. Mod Pathol 2010;23:1191-200.

29. Nikiforov YE. Role of molecular markers in thyroid nodule management: then and now. Endocr Pract 2017;23:979-88.

30. Ohba K, Mitsutake N, Matsuse M, et al. Encapsulated Papillary Thyroid Tumour with Delicate Nuclear Changes and a KRAS Mutation as a Possible Novel Subtype of Borderline Tumour. J Pathol Transl Med 2019;53:136-41.

31. Rosario PW. Noninvasive encapsulated papillary RAS-like thyroid tumour (NEPRAS) or encapsulated 
papillary thyroid carcinoma (PTC). J Pathol Transl Med 2020;54:263-4.

32. Liu Z, Zhou G, Nakamura M, et al. Encapsulated follicular thyroid tumour with equivocal nuclear changes, socalled well-differentiated tumour of uncertain malignant potential: a morphological, immunohistochemical, and molecular appraisal. Cancer Sci 2011;102:288-94.

33. Cameselle-Teijeiro JM, Sobrinho-Simões $M$. New WHO classification of thyroid tumours: a pragmatic categorization of thyroid gland neoplasms. Endocrinol Diabetes Nutr 2018;65:133-5.

34. Canberk S, Baloch Z, Ince U, et al. Diagnosis of noninvasive follicular tumour with papillary-like nuclear features (NIFTP): a practice changer for thyroid fineneedle aspiration interpretation. J Basic Clin Med 2017;6:38-43.

35. Kim MJ, Won JK, Jung KC, et al. Clinical characteristics of subtypes of follicular variant papillary thyroid carcinoma. Thyroid 2018;28:311-8.

36. Parente DN, Kluijfhout WP, Bongers PJ, et al. Clinical safety of renaming encapsulated follicular variant of papillary thyroid carcinoma: is NIFTP truly benign? World J Surg 2018;42:321-6.

37. Cho U, Mete O, Kim MH, et al. Molecular correlates and rate of lymph node metastasis of non-invasive follicular thyroid neoplasm with papillary-like nuclear features and invasive follicular variant papillary thyroid carcinoma: the impact of rigid criteria to distinguish non-invasive follicular thyroid neoplasm with papillary-like nuclear features. Mod Pathol 2017;30:810-25.

38. Lloyd RV, Asa S, LiVolsi V, et al. The evolving diagnosis of noninvasive follicular thyroid neoplasm with papillarylike nuclear features (NIFTP). Hum Pathol 2018;74:1-4.

39. Alves VAF, Kakudo K, LiVolsi V, et al. Noninvasive Follicular Thyroid Neoplasm with Papillary-Like Nuclear Features (NIFTP): Achieving Better Agreement By Refining Diagnostic Criteria. Clinics (Sao Paulo) 2018;73:e576.

40. Thompson LDR, Poller DN, Kakudo K, et al. An international interobserver variability reporting of the nuclear scoring criteria to diagnose noninvasive follicular thyroid neoplasm with papillary-like nuclear features: A validation study. Endocr Pathol 2018;29:242-9.

41. Liu Z, Bychkov A, Jung CK, et al. Interobserver and intraobserver variation in the morphological evaluation of noninvasive follicular thyroid neoplasm with papillarylike nuclear features in Asian practice. Pathol Int 2019;69:202-10.
42. Bychkov A, Hirokawa M, Jung CK, et al. Low Rate of Noninvasive Follicular Thyroid Neoplasm with PapillaryLike Nuclear Features in Asian Practice. Thyroid 2017;27:983-4.

43. Vuong HG, Ngo HTT, Bychkov A, et al. Differences in surgical resection rate and risk of malignancy in thyroid cytopathology practice between Western and Asian countries: A systematic reviewand meta-analysis. Cancer Cytopathol 2020;128:238-49.

44. Baloch ZW, Harrell RM, Brett EM, et al. American Association of Clinical Endocrinologists and American College of Endocrinology disease state commentary: managing thyroid tumours diagnosed as noninvasive follicular thyroid neoplasm with papillary-like nuclear features. Endocr Pract 2017;23:1150-5.

45. Canberk S, Lima AR, Correia M, et al. Oncocytic thyroid neoplasms: from histology to molecular biology. Diagn Histopathol 2019;25:154-65.

46. Ali SZ, Cibas ES. The Bethesda System for Reporting Thyroid Cytopathology: Definitions, Criteria, and Explanatory Notes. vol. 2. New York, NY: Springer; 2018.

47. Canberk S, Gunes P, Onenerk M, et al. New concept of the encapsulated follicular variant of papillary thyroid carcinoma and its impact on the Bethesda system for reporting thyroid cytopathology: a single- institute experience. Acta Cytol 2016;60:198-204.

48. Faquin WC, Wong LQ, Afrogheh AH, et al. Impact of reclassifying noninvasive follicular variant of papillary thyroid carcinoma on the risk of malignancy in the Bethesda system for reporting thyroid cyto- pathology. Cancer Cytopathol 2016;124:181-7.

49. Strickland KC, Howitt BE, Marqusee E, et al. The impact of noninva- sive follicular variant of papillary thyroid carcinoma on rates of malig- nancy for fine-needle aspiration diagnostic categories. Thyroid 2015;25:987-92.

50. Seethala RR, Baloch ZW, Barletta JA, et al. Noninvasive follicular thyroid neoplasm with papillary-like nuclear features: a review for pathologists. Mod Pathol 2018;31:39-55.

51. Rana C, Manjunath S, Ramakant $\mathrm{P}$, et al. Noninvasive follicular neoplasm with papillary like nuclear features: A comprehensive analysis with a diagnostic algorithm. Diagn Cytopathol 2020;48:330-41.

52. Liu Z, Kakudo K, Zhang C, et al. Biological Behaviour of Thyroid Cancer in Indeterminate Category. J Basic Clin Med 2015;4:87-91.

53. Chetty R. Follicular patterned lesions of the thyroid gland: a practical algorithmic approach. J Clin Pathol 
2011;64:737-41.

54. Fusco A, Chiappetta G, Hui P, et al. Assessment of RET/ PTC oncogene activation and clonality in thyroid nodules with incomplete morphological evidence of papillary carcinoma: a search for the early precursors of papillary cancer. Am J Pathol 2002;160:2157-67.

55. Kakudo K, Bai Y, Liu Z, et al. Classification of thyroid follicular cell tumours: with special reference to borderline lesions. Endocr J 2012;59:1-12.

56. Duan H, Liu X, Ren X, et al. Mutation profiles of

Cite this article as: Canberk S. Precursor and borderline lesions of the thyroid (indolent lesions of epithelial origin): from theory to practice. Gland Surg 2020;9(5):1724-1734. doi: 10.21037/gs-20429 follicular thyroid tumours by targeted sequencing. Diagn Pathol 2019;14:39.

57. Zhu Y, Li Y, Jung CK, et al. Histopathologic Assessment of Capsular Invasion in Follicular Thyroid Neoplasms - an Observer Variation Study. Endocr Pathol 2020;31:132-40.

58. Paulsson JO, Mu N, Shabo I, et al. TERT aberrancies: a screening tool for malignancy in follicular thyroid tumours. Endocr Relat Cancer 2018;25:723-33.

(English Language Editor: J. Chapnick) 